\title{
Stellar population gradients in brightest cluster galaxies
}

\author{
S. I. Loubser ${ }^{1}$ and P. Sánchez-Blázquez ${ }^{2}$ \\ ${ }^{1}$ Centre for Space Research, North-West University, Potchefstroom 2520, South Africa \\ email: Ilani.Loubser@nwu.ac.za \\ ${ }^{2}$ Departamento de Física Teórica, Universidad Autónoma de Madrid, E28049, Spain \\ email: psanchezblazquez@googlemail.com
}

\begin{abstract}
We present the stellar population and velocity dispersion gradients for a sample of 24 brightest cluster galaxies (BCGs) in the nearby Universe for which we have obtained high quality long-slit spectra at the Gemini telescopes. With the aim of studying the possible connection between the formation of the BCGs and their host clusters, we explore the relations between the stellar population gradients and properties of the host clusters, as well as the possible connections between the stellar population gradients and other properties of the galaxies. We find mean stellar population gradients (negative $\Delta[\mathrm{Z} / \mathrm{H}] / \log r$ gradient of $-0.285 \pm 0.064$; small positive $\Delta \log ($ age $) / \log r$ gradient of $+0.069 \pm 0.049$; and null $\Delta[\mathrm{E} / \mathrm{Fe}] / \log r$ gradient of $-0.008 \pm 0.032)$, that are consistent with those of normal massive elliptical galaxies. However, we find a trend between metallicity gradients and velocity dispersion (with a negative slope of $-1.616 \pm 0.539$ ), that is not found for the most massive ellipticals. Furthermore, we find trends between the metallicity gradients and $K$-band luminosities (with a slope of $0.173 \pm 0.081$ ) as well as the distance from the BCG to the X-ray peak of the host cluster (with a slope of $-7.546 \pm 2.752$ ). The latter indicates a possible relation between the formation of the cluster and that of the central galaxy.
\end{abstract}

Keywords. galaxies: evolution - galaxies: elliptical and lenticular, $\mathrm{cD}$ - galaxies: stellar content

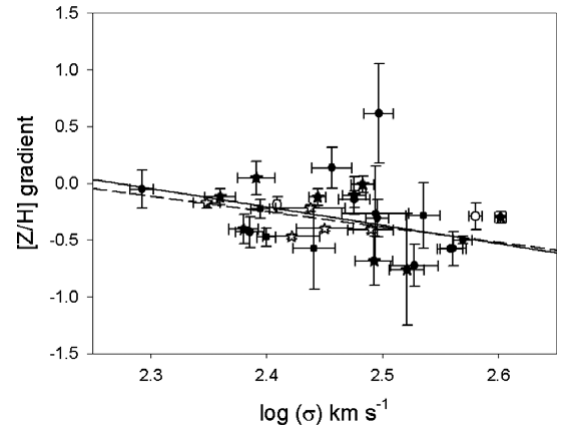

Figure 1. Metallicity gradients plotted against central velocity dispersion. The BCGs in our sample are shown with black symbols (those observed within 15 degrees of the MA are indicated with stars, and the others with circles. The BCGs from other studies are shown with empty symbols (see Loubser \& Sánchez-Blázquez 2012). The fitted metallicity - mass correlation for our sample is shown with a solid line, and the correlation fitted to all the data with a dashed line.

\section{Reference}

Loubser, S. I. \& Sánchez-Blázquez, P. 2012, MNRAS, 425, 841 